\title{
Nucleon form factors with dynamical twisted mass fermions
}

\section{Constantia Alexandrou,Tomasz Korzec*, Giannis Koutsou}

Department of Physics, University of Cyprus, P.O. Box 20537, 1678 Nicosia, Cyprus

E-mail: alexand@ucy.ac.cy, korzec@ucy.ac.cy, koutsou@ucy.ac.cy

Mariane Brinet, Jaume Carbonell, Vincent Drach, Pierre-Antoine Harraud

Laboratoire de Physique Subatomique et Cosmologie, 53 avenue des Martyrs, 38026 Grenoble,

France

E-mail: marianedlpsc.in2p3.fr, carbonelelpsc.in2p3.fr,

drachelpsc.in2p3.fr harraudelpsc.in2p3.fr

\section{Rémi Baron}

CEA, Centre de Saclay, IRFU/Service de Physique Nucléaire, F-91191 Gif-sur-Yvette, France

E-mail: remi.baronecea.fr

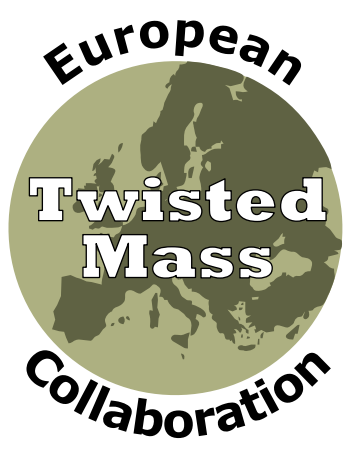

The electromagnetic and axial form factors of the nucleon are evaluated in twisted mass QCD with two degenerate flavors of light, dynamical quarks. The axial charge $g_{A}$, magnetic moment and the Dirac and Pauli radii are determined for pion masses in the range $300 \mathrm{MeV}$ to $500 \mathrm{MeV}$.

The XXVI International Symposium on Lattice Field Theory

July 14 - 19, 2008

Williamsburg, Virginia, USA

\footnotetext{
* Speaker.
} 


\section{Introduction}

Recent calculations in both the meson [1, 2] and baryon [3] sectors within the twisted mass formulation of lattice QCD [ [ 4 ] have yielded accurate results on a wide range of observables of immediate relevance to experiment and phenomenology [5]. This is a consequence of the fact that the simulations of the European Twisted Mass Collaboration (ETMC) cover a well chosen range of parameters, allowing controlled continuum and chiral extrapolations and thus making a reliable connection to the physical regime possible. In the present work we apply the twisted mass framework to the calculation of nucleon form factors.

Electromagnetic and axial form factors of the proton and the neutron are fundamental quantities that yield information on their internal structure such as their size, magnetization and axial charge. They have been studied experimentally for a long time with steadily increasing precision, the latest generation of experiments reaching relative precisions of down to about $1 \%$ [6]. The first lattice calculations using dynamical fermions appeared only recently [7, 8, 9] and the available pion masses were rather large, often above $400 \mathrm{MeV}$.

Our goal is to calculate matrix elements of the form $\left\langle N\left(p_{f}, s_{f}\right)\left|\mathscr{O}_{\mu}\right| N\left(p_{i}, s_{i}\right)\right\rangle$, where $|N(p, s)\rangle$ is the nucleon ground state, with the nucleon having momentum $p$ and spin $s$. $\mathscr{O}_{\mu}$ is either the electromagnetic or the axial current. Using, in addition, the pseudoscalar current will enable us to check phenomenological consequences of chiral symmetry such as the Goldberger-Treiman relation [10]. The electromagnetic matrix element can be expressed in terms of two Lorentz invariant form factors that depend on the momentum transfer squared only. In Euclidean space-time the decomposition is

$$
\left\langle N\left(p_{f}, s_{f}\right)\left|V_{\mu}(0)\right| N\left(p_{i}, s_{i}\right)\right\rangle=\bar{u}\left(p_{f}, s_{f}\right)\left[\gamma_{\mu} F_{1}\left(Q^{2}\right)+\frac{\sigma_{\mu \nu} Q_{v}}{2 m} F_{2}\left(Q^{2}\right)\right] u\left(p_{i}, s_{i}\right),
$$

where $Q=p_{f}-p_{i}, \sigma_{\mu v}=i\left[\gamma_{\mu}, \gamma_{v}\right] / 2, m$ is the proton mass and $u(p, s)$ is a solution to the free Dirac equation with mass $m$. Instead of the Dirac and Pauli form factors $F_{1}$ and $F_{2}$ the matrix element can be expressed in terms of the electric and magnetic Sachs form factors

$$
G_{E}=F_{1}\left(Q^{2}\right)-\frac{Q^{2}}{4 m^{2}} F_{2}\left(Q^{2}\right), \quad G_{M}=F_{1}\left(Q^{2}\right)+F_{2}\left(Q^{2}\right) .
$$

Similarly the axial current matrix element can be written in terms of the form factors $G_{A}$ and $G_{p}$,

$$
\left\langle N\left(p_{f}, s_{f}\right)\left|A_{\mu}(0)\right| N\left(p_{i}, s_{i}\right)\right\rangle=\bar{u}\left(p_{f}, s_{f}\right)\left[\gamma_{5} \gamma_{\mu} G_{A}\left(Q^{2}\right)+\frac{i Q_{\mu} \gamma_{5}}{2 m} G_{p}\left(Q^{2}\right)\right] u\left(p_{i}, s_{i}\right) .
$$

\section{Calculation details}

\subsection{Wilson twisted mass QCD}

For our calculation we use a Wilson twisted mass fermion action (tmQCD) at maximal twist angle together with a tree-level Symanzik improved gauge action. More precisely, the fermion action is given by

$$
S^{f}=a^{4} \sum_{x} \bar{\chi}\left[\frac{1}{2}\left(\gamma_{\mu}\left(\nabla_{\mu}+\nabla_{\mu}^{*}\right)-a \nabla_{\mu} \nabla_{\mu}^{*}\right)+m_{\mathrm{crit}}+i \gamma_{5} \tau_{3} \mu\right] \chi
$$


with lattice spacing $a$, the covariant forward and backward lattice derivatives $\nabla$ and $\nabla^{*}$ and mass parameters $m_{\text {crit }}$ and $\mu$. The Pauli matrix $\tau_{3}$ acts on a doublet $\chi^{\top}=(u, d)$ of two light quarks. Automatic $O(a)$ improvement occurs at maximal twist [11], which here is realized by tuning the value of $m_{\text {crit }}$ so that the PCAC mass vanishes. The gauge action is

$$
S^{g}=\frac{\beta}{3} \sum_{x}\left(\frac{5}{3} \sum_{\mu, v>\mu}\left(1-\operatorname{Re} \operatorname{Tr}\left[U_{x, \mu, v}^{1 \times 1}\right]\right)-\frac{1}{12} \sum_{\mu, v \neq \mu}\left(1-\operatorname{Re} \operatorname{Tr}\left[U_{x, \mu, v}^{1 \times 2}\right]\right)\right),
$$

where $U_{x, \mu, v}^{1 \times 1}$ denotes the plaquette term, $U_{x, \mu, v}^{1 \times 2}$ the rectangular $1 \times 2$ Wilson loops and $\beta$ is the inverse bare coupling. Further details concerning the simulation and in particular the tuning to maximal twist can be found in Ref. [12].

\subsection{Correlation functions}

On the lattice the form factors are extracted from dimensionless ratios of correlation functions. We measure the two- and three- point functions

$$
\begin{aligned}
G(\vec{q}, t) & =\sum_{\vec{x}_{f}} e^{-i \vec{x}_{f} \cdot \vec{q}} \Gamma_{\beta \alpha}^{0}\left\langle J_{\alpha}\left(t_{f}, \vec{x}_{f}\right) \bar{J}_{\beta}(0)\right\rangle, \\
G_{\mu}\left(\Gamma^{v}, \vec{q}, t\right) & =\sum_{\vec{x}, \vec{x}_{f}} e^{i \vec{x} \cdot \vec{q}} \Gamma_{\beta \alpha}^{v}\left\langle J_{\alpha}\left(t_{f}, \vec{x}_{f}\right) \mathscr{O}_{\mu}(t, \vec{x}) \bar{J}_{\beta}(0)\right\rangle,
\end{aligned}
$$

where $\Gamma^{v}=1 / 4\left[\mathbb{1}+\gamma_{0}\right]$ if $v=0$ and $\Gamma^{v}=1 / 4\left[\mathbb{1}+\gamma_{0}\right] \gamma_{5} \gamma_{v}$ if $v=1,2$ or 3 . and $J$ is the proton interpolating field. In tmQCD at maximal twist the standard interpolating field reads

$$
J(x)=\frac{1}{\sqrt{2}}\left[\mathbb{1}+i \gamma_{5}\right] \varepsilon^{a b c}\left[\tilde{u}^{a \top}(x) \mathscr{C} \gamma_{5} \tilde{d}^{b}(x)\right] \tilde{u}^{c}(x),
$$

with $\mathscr{C}$ denoting the charge conjugation matrix. To enhance the overlap of $J$ with the proton ground state, the quark fields entering Eq. (2.5) are smeared,

$$
\begin{gathered}
\tilde{u}^{a}(t, \vec{x})=\sum_{\vec{y}} F^{a b}(\vec{x}, \vec{y} ; U(t)) u^{b}(t, \vec{y}), \\
F=(\mathbb{1}+\alpha H)^{N}, \quad H(\vec{x}, \vec{y} ; U(t))=\sum_{i=1}^{3}\left(U_{i}(x) \delta_{x, y-\hat{\imath}}+U_{i}^{\dagger}(x-\hat{\imath}) \delta_{x, y+\hat{\imath}}\right) .
\end{gathered}
$$

In addition, the spatial gauge links entering the hopping matrix $H$ are APE-smeared. Good choices for the parameters $\alpha$ and $N$ were determined in Ref. [3].

In this work, we restrict ourselves to the axial-vector and vector currents for the operators $\mathscr{O}_{\mu}$ inserted into the three point functions. We use the local currents

$$
\begin{aligned}
A_{\mu}(x) & =\bar{u} \gamma_{\mu} \gamma_{5} u-\bar{d} \gamma_{\mu} \gamma_{5} d \\
V_{\mu}(x) & =\bar{u} \gamma_{\mu} u-\bar{d} \gamma_{\mu} \gamma_{5} d,
\end{aligned}
$$

as well as the symmetrized, conserved vector current

$$
\begin{aligned}
V_{\mu}^{N}(x) & =\frac{1}{2}\left[j_{\mu}^{u}(x)+j_{\mu}^{u}(x-\hat{\mu})\right]-\frac{1}{2}\left[j_{\mu}^{d}(x)+j_{\mu}^{d}(x-\hat{\mu})\right] \quad \text { where } \\
j_{\mu}^{q}(x) & =\bar{q}(x+\hat{\mu}) \frac{1}{2}\left[\gamma_{\mu}+\mathbb{1}\right] U_{\mu}^{-1}(x) q(x)+\bar{q}(x) \frac{1}{2}\left[\gamma_{\mu}-\mathbb{1}\right] U_{\mu}(x) q(x+\hat{\mu}) .
\end{aligned}
$$


While the two-point functions can be calculated using a forward propagator from a fixed source, the evaluation of three-point functions is more involved. In correlators containing the isovector operators, disconnected diagrams are zero up to lattice artifacts, and can be safely neglected as we approach the continuum limit. The connected diagrams are calculated using sequential inversions through the sink [13]. This means that at a fixed source-sink separation we are able to obtain results for all possible momentum transfers and insertion times as well as for any operator $\mathscr{O}_{\mu}$, with a two sequential inversions per choice of the sink. In this work we use two different sinks, one optimized for the electric and one for the magnetic form factor [7]. The latter is also suitable for the axial form factors.

\subsection{Ratios}

In ratios of three- and two- point functions normalization factors and the leading exponential dependencies on the insertion time cancel. From fits to the resulting plateaus, the form factors can be extracted. Different ratios can be considered and we here give two examples:

$$
R^{\mu}(\Gamma, \vec{q}, t)=\frac{G^{\mu}(\Gamma, \vec{q}, t)}{\sqrt{G\left(\overrightarrow{0}, 2\left(t_{f}-t\right)\right) G\left(\vec{q}, 2\left(t-t_{i}\right)\right)}}
$$

or

$$
R^{\mu}(\Gamma, \vec{q}, t)=\frac{G^{\mu}(\Gamma, \vec{q}, t)}{G\left(\overrightarrow{0}, t_{f}\right)} \sqrt{\frac{G\left(\vec{q}, t_{f}-t\right) G(\overrightarrow{0}, t) G\left(\overrightarrow{0}, t_{f}\right)}{G\left(\overrightarrow{0}, t_{f}-t\right) G(\vec{q}, t) G\left(\vec{q}, t_{f}\right)}} .
$$

As shown in Fig. 1, both lead to compatible plateaus but to different statistical errors. We use
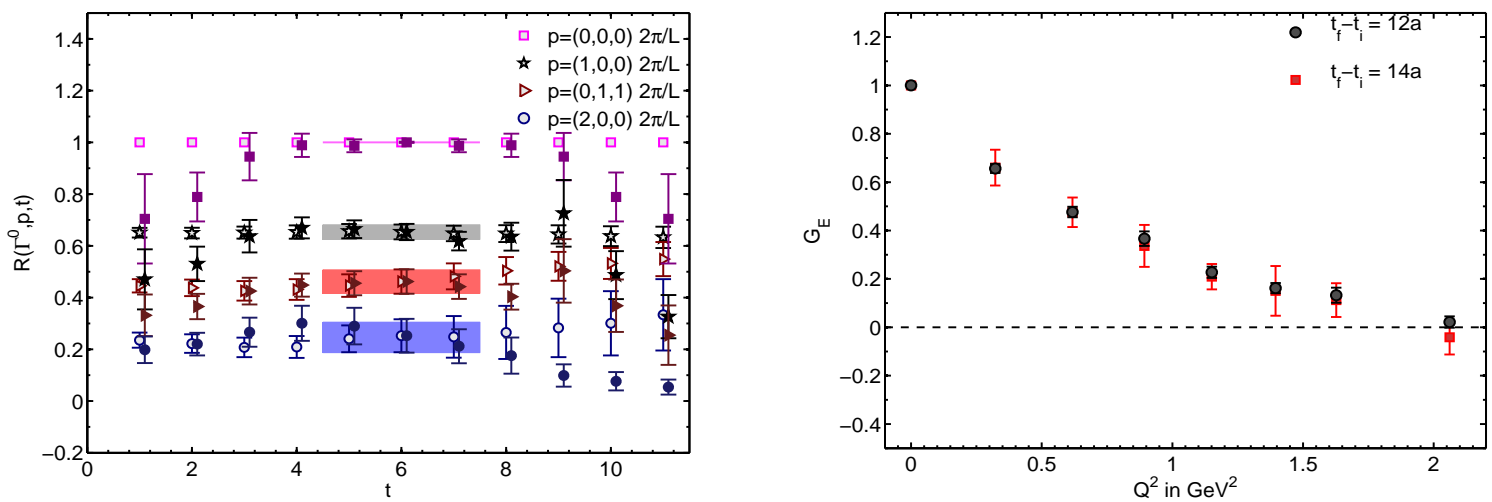

Figure 1: Left panel: Comparison of the ratios given in Eqs. (2.10) (filled symbols) and (2.11) (open symbols) for source type given in Eq. (2.13) and a few representative momentum combinations. Right panel: Electric Sachs form factor extracted with a sink source separation of $12 a$ and $14 a$.

the ratio of Eq. (2.11) for the final analysis, which turns out to be superior because it does not contain potentially noisy two point functions at large separations and because correlations between its different factors reduce the statistical noise. This being most evident at zero momentum with the conserved current, where noise cancels completely.

Once the plateau values

$$
\Pi(\Gamma, \vec{q})=\lim _{t_{f}-t \rightarrow \infty} \lim _{t-t_{i} \rightarrow \infty} R(\Gamma, \vec{q}, t)
$$


are estimated, the form factors are obtained from the following combinations

$$
\begin{aligned}
\Pi^{\mu}\left(\Gamma^{0}, \vec{q}\right) & =\frac{c}{2 m}\left[(m+E) \delta_{0, \mu}+\sum_{k} i q_{k} \delta_{k, \mu}\right] G_{E}\left(Q^{2}\right) \\
\Pi^{i}\left(\Gamma^{1}, \vec{q}\right)+\Pi^{i}\left(\Gamma^{2}, \vec{q}\right)+\Pi^{i}\left(\Gamma^{3}, \vec{q}\right) & =\frac{c}{2 m} \sum_{j k l} \varepsilon_{j k l} q_{j} \delta_{l, i} G_{M}\left(Q^{2}\right) \\
\Pi^{5 i}\left(\Gamma^{1}, \vec{q}\right)+\Pi^{5 i}\left(\Gamma^{2}, \vec{q}\right)+\Pi^{5 i}\left(\Gamma^{3}, \vec{q}\right) & =\frac{i c}{4 m}\left[\left(q_{1}+q_{2}+q_{3}\right) \frac{q_{i}}{2 m} G_{p}\left(Q^{2}\right)-(E+m) G_{A}\left(Q^{2}\right)\right] .
\end{aligned}
$$

$E$ is the energy of a proton with momentum $\vec{p}_{i}, m$ its mass and the constant $c=\sqrt{\frac{2 m^{2}}{E(E+m)}}$ arises from the normalization of lattice hadron states that we use. Note that the sequential source needed in the calculations of the magnetic Sachs form factor is the same as the one for $G_{A}$ and $G_{p}$.

\section{Results}

We perform the calculation at three pion masses with a fixed lattice spacing. The source and sink time slices are taken at $t_{i}=0$ and $t_{f}=12$ and a check with $t_{f}-t_{i}=14$ on 96 configurations at the highest mass gave compatible results, as shown in the right panel of Fig. 1. The remaining parameters of the calculation together with the most important results are summarized in Table. 1.

\begin{tabular}{ccccccc}
\hline$m_{\pi}[\mathrm{GeV}]$ & number of confs & $m_{N}[\mathrm{GeV}]$ & $\sqrt{\left\langle r_{1}^{2}\right\rangle}[\mathrm{fm}]$ & $\sqrt{\left\langle r_{2}^{2}\right\rangle}[\mathrm{fm}]$ & $\mu_{I V}\left[\mu_{N}\right]$ & $g_{A}$ \\
\hline $0.4470(12)$ & 346 & $1.287(13)$ & $0.489(25)$ & $0.558(61)$ & $2.83(21)$ & $1.171(41)$ \\
$0.3903(9)$ & 184 & $1.245(9)$ & $0.527(34)$ & $0.607(79)$ & $2.90(36)$ & $1.096(47)$ \\
$0.3131(16)$ & 419 & $1.143(11)$ & $0.649(38)$ & $0.63(11)$ & $2.85(46)$ & $1.23(10)$ \\
\hline
\end{tabular}

Table 1: The form factors are extracted using a lattice of size $24^{3} \times 48$ at $\beta=3.9$ with a lattice spacing of $a=0.0889(12)$. This table summarizes the main results at the three different pion masses.

\subsection{Electric and magnetic Sachs form factors}

The dependence of $G_{E}$ and $G_{M}$ on the euclidean momentum transfer squared is shown in Fig. 2. A dipole form

$$
G_{E}\left(Q^{2}\right)=\frac{1}{\left(1+\frac{Q^{2}}{m_{E}^{2}}\right)^{2}}, \quad G_{M}\left(Q^{2}\right)=\frac{G_{M}(0)}{\left(1+\frac{Q^{2}}{m_{M}^{2}}\right)^{2}}
$$

describes our data very well and the solid lines in Fig. 2 are the corresponding least squares fits. From the slope at zero momentum transfer, an electric and magnetic radius can be defined

$$
\left\langle r_{E, M}^{2}\right\rangle=-\left.\frac{6}{G_{E, M}\left(Q^{2}\right)} \frac{d G_{E, M}\left(Q^{2}\right)}{d Q^{2}}\right|_{Q^{2}=0}=\frac{12}{m_{E, M}^{2}} .
$$

These can easily be translated into the more common Dirac and Pauli radii

$$
\left\langle r_{1}^{2}\right\rangle=\frac{12}{m_{E}^{2}}-\frac{3 G_{M}(0)-3}{2 m^{2}}, \quad\left\langle r_{2}^{2}\right\rangle=\frac{6}{G_{M}(0)-1}\left(\frac{2 G_{M}(0)}{m_{M}^{2}}-\frac{2}{m_{E}^{2}}\right)+\frac{3}{2 m^{2}} .
$$



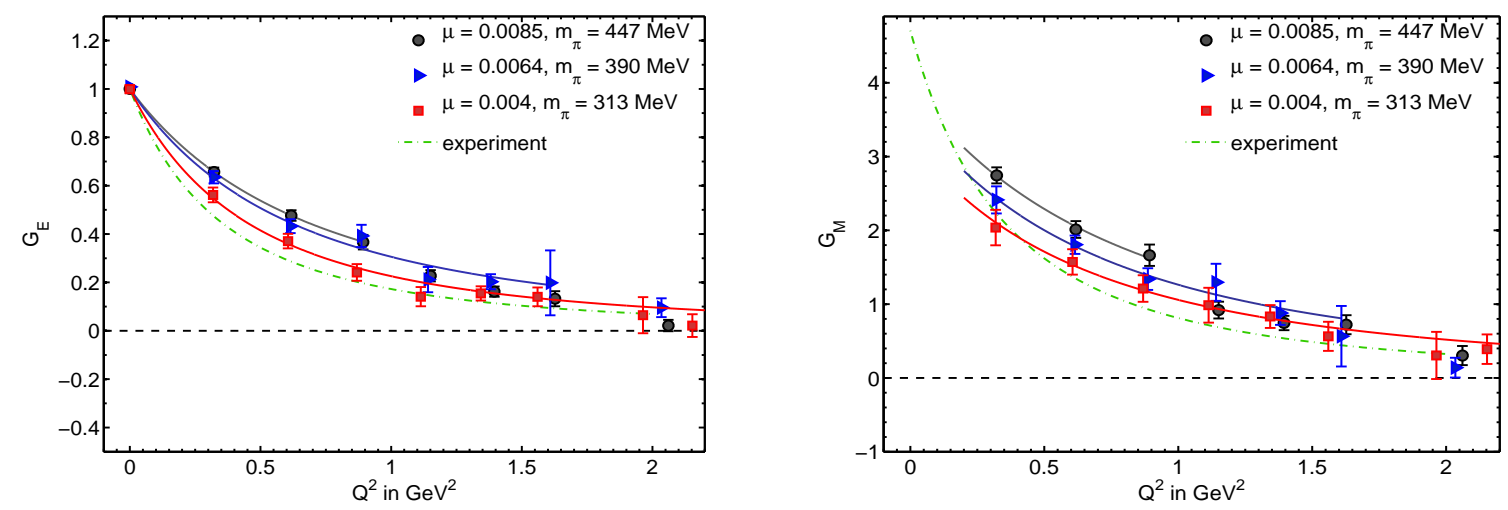

Figure 2: $Q^{2}$-dependence of $G_{E}$ (left) and $G_{M}$ (right). The dashed curve is a dipole fit to experimental data.
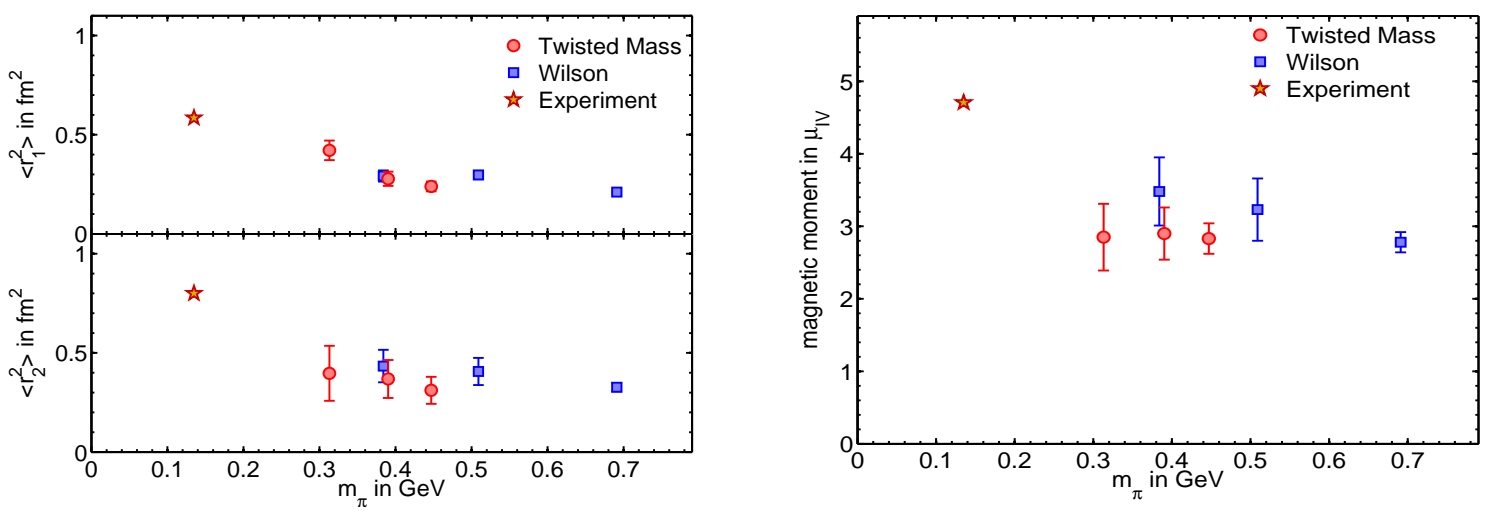

Figure 3: Comparison of results on $\left\langle r_{1}^{2}\right\rangle,\left\langle r_{2}^{2}\right\rangle$ and $\mu_{I V}$ between $N_{F}=2$ twisted mass and Wilson fermions.

Using the value of the lattice spacing determined from an analysis of the nucleon mass within the twisted mass QCD framework we convert our lattice quantities to physical units and give the results in Table 1. The magnetic form factor at zero momentum transfer is directly related to the magnetic moment, $G_{M}(0) \frac{e \hbar}{2 m_{N}}=\mu_{I V}$. In Table 11 we give the isovector magnetic moment converted to nuclear magnetons using the physical nucleon mass. We show the dependence of $\left\langle r_{1}^{2}\right\rangle,\left\langle r_{2}^{2}\right\rangle$ and $\mu_{I V}$ on the pion mass in Fig. 3. For comparison we include the corresponding results of Ref. [7], which were obtained with two degenerate dynamical Wilson fermions in a similar setup. As can be seen, the results in the two formulations are in good agreement. With the isovector current, the corresponding physical observable is the magnetic moment of the proton minus the one of the neutron. This is the experimental point shown in the right panel of Fig. 3. As can be seen, the experimental values in all cases are higher than lattice results. A chiral extrapolation of our data to the physical point will be carried out once we obtain results at an additional lighter pion mass. This additional input is needed in order to obtain reliable results at the physical point.

\subsection{Axial form factors}

The axial form factors are shown in Fig. \# with $G_{p}$ showing the larger statistical errors. We get a good signal for the axial charge $g_{A}=Z_{A} G_{A}(0)$, with the renormalization constant $Z_{A}=0.76(1)$ that has been computed in [14]. We give our values in Table. 1. 

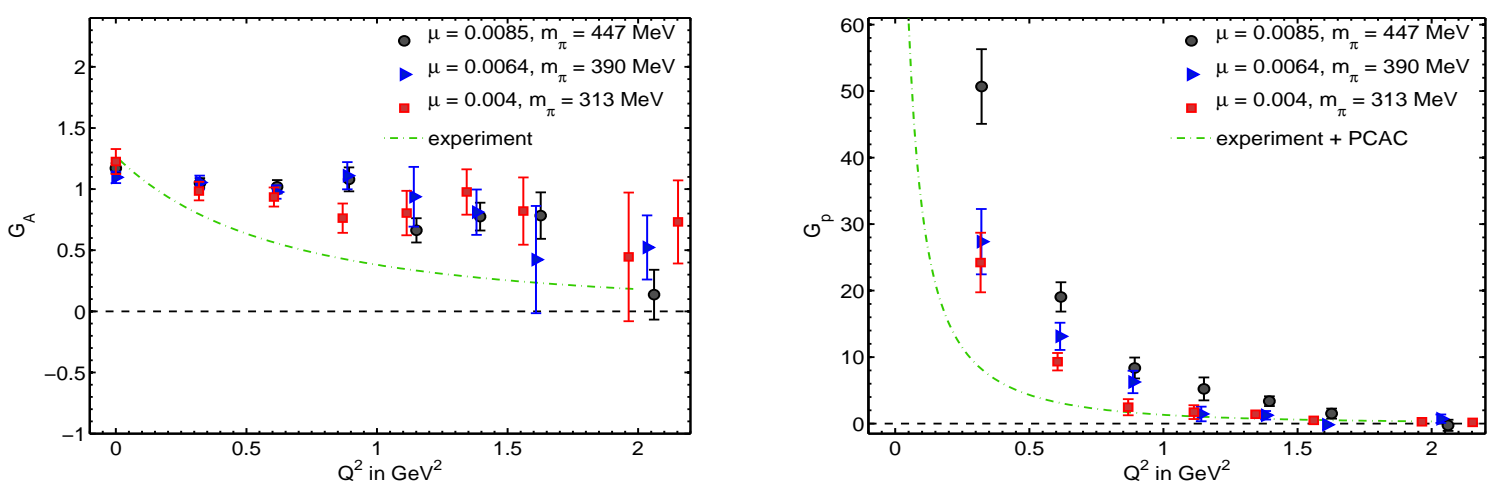

Figure 4: $Q^{2}$-dependence of $G_{A}$ (left) and $G_{p}$ (right). The dashed green curve (left) is a dipole fit to experimental data on $G_{A}$ yielding $m_{A}=1.1 \mathrm{GeV}$. In the right figure it derives from the fit to experimental results on $G_{A}$ together with the assumption of pion pole dominance.

\section{Conclusions}

The isovector electromagnetic and axial nucleon form factors are evaluated using two dynamical degenerate twisted mass fermions for pion masses down to about $300 \mathrm{MeV}$. The results are in agreement with previous lattice studies [7]. Like in Ref. [7], we find better agreement with experiment in the case of the electric form factor than for the magnetic one. Although our results on the axial charge are in good agreement with experiment, we find a weaker momentum dependence for $G_{A}$. At low $Q^{2}, G_{p}$ shows a steep increase as expected from pion pole dominance and approaches the theoretical prediction as the pion mass decreases. In the future we plan to analyze configurations at a lighter pion mass and larger volume. This will enable us to reliably carry out a chiral extrapolation of the magnetic moment, radii and axial charge to the physical point.

\section{References}

[1] P. Boucaud et al., Phys. Lett. B650 (2007) 304, hep-lat/0701012.

[2] K. Jansen, C. Michael and C. Urbach, (2008), arXiv:0804.3871.

[3] C. Alexandrou et al., Phys. Rev. D78 (2008) 014509, arXiv:0803.3190.

[4] Alpha, R. Frezzotti et al., JHEP 08 (2001) 058, hep-lat/0101001.

[5] H. Leutwyler, (2007), arXiv:0706.3138 [hep-ph].

[6] V. Punjabi et al., Phys. Rev. C71 (2005) 055202, nucl-ex/0501018.

[7] C. Alexandrou et al., Phys. Rev. D74 (2006) 034508, hep-lat/0605017.

[8] M. Gockeler et al., (2007), arXiv0709.3370.

[9] P. Hagler et al., Phys. Rev. D77 (2008) 094502, arXiv:0705.4295.

[10] C. Alexandrou et al., Phys. Rev. D76 (2007) 094511.

[11] R. Frezzotti and G.C. Rossi, JHEP 08 (2004) 007, hep-lat/0306014.

[12] P. Boucaud et al., (2008), arXiv:0803.0224.

[13] D. Dolgov et al., Phys. Rev. D66 (2002) 034506, hep-lat/0201021.

[14] P. Dimopoulos et al., PoS LAT2007 (2007) 241, arXiv:0710.0975. 